\title{
Aeroacoustic Simulation of a Nose Landing Gear in an Open Jet Facility using FUN3D
}

\author{
Veer N. Vatsa, ${ }^{*}$ David P. Lockard, ${ }^{\dagger}$ Mehdi R. Khorrami, ${ }^{*}$ and Jan-Renee Carlson ${ }^{\dagger}$ \\ NASA Langley Research Center, Hampton, VA 23681
}

\begin{abstract}
Numerical simulations have been performed for a partially-dressed, cavity-closed nose landing gear configuration that was tested in NASA Langley's closed-wall Basic Aerodynamic Research Tunnel (BART) and in the University of Florida's open-jet acoustic facility known as UFAFF. The unstructured-grid flow solver, FUN3D, developed at NASA Langley Research center is used to compute the unsteady flow field for this configuration. A hybrid Reynolds-averaged Navier-Stokes/large eddy simulation (RANS/LES) turbulence model is used for these computations. Time-averaged and instantaneous solutions compare favorably with the measured data. Unsteady flowfield data obtained from the FUN3D code are used as input to a Ffowcs Williams-Hawkings noise propagation code to compute the sound pressure levels at microphones placed in the farfield. Significant improvement in predicted noise levels is obtained when the flowfield data from the open jet UFAFF simulations is used as compared to the case using flowfield data from the closed-wall BART configuration.
\end{abstract}

\section{Nomenclature}

$\begin{array}{ll}\text { BART } & \text { Basic Aerodynamic Research Tunnel } \\ \text { BDF } & \text { backward differencing formulation } \\ \text { BDF2 } & \text { second-order backward differencing formulation } \\ \text { BDF2OPT } & \text { optimized second-order backward differencing formulation } \\ \text { CAA } & \text { computational aeroacoustics } \\ \text { CFD } & \text { computational fluid dynamics } \\ C_{p} & \left(p-p_{\infty}\right) /\left(0.5 \rho_{\infty} u_{\infty}^{2}\right) \\ \text { DES } & \text { detached eddy simulation } \\ \text { DDES } & \text { delayed detached eddy simulation } \\ \text { FW-H } & \text { Ffowcs Williams-Hawkings } \\ \text { HRLES } & \text { hybrid RANS/LES } \\ \text { LES } & \text { large eddy simulation } \\ \text { MDDES } & \text { modified delayed detached eddy simulation } \\ \text { NLG } & \text { nose landing gear } \\ \text { PDCC } & \text { partially-dressed, cavity-closed } \\ \text { PIV } & \text { particle image velocimetry } \\ \text { psi } & \text { pound per square inch } \\ \text { PSD } & \text { power spectral density } \\ r & \text { radial distance } \\ \text { RANS } & \text { Reynolds-averaged Navier-Stokes } \\ \text { SPL } & \text { sound pressure level } \\ \text { SST } & \text { shear stress transport } \\ \text { TKE } & \text { turbulence kinetic energy } \\ \text { UFAFF } & \text { University of Florida Aeroacoustic Flow Facility } \\ \text { URANS } & \text { unsteady Reynolds-averaged Navier-Stokes } \\ u, v, w & \text { Cartesian fluid velocity components } \\ X, Y, Z & \text { Cartesian coordinates } \\ \theta & \text { circumferential angle } \\ \rho & \text { density } \\ & \end{array}$

\footnotetext{
*Aerospace Engineer, Computational AeroSciences Branch, Research Directorate; Associate Fellow AIAA

${ }^{\dagger}$ Aerospace Engineer, Computational AeroSciences Branch, Research Directorate; Senior Member AIAA
} 


\author{
Superscript: \\ , perturbation quantity (e.g. $\left.u^{\prime}=u-u_{\infty}\right)$ \\ Subscript: \\ $\infty$ \\ free-stream quantity
}

\title{
I. Introduction
}

In recent years, there has been growing emphasis on reducing airframe noise to meet the increasingly restrictive standards for permissible noise near airports. The landing gear is a significant contributor to airframe noise during approach and landing for commercial aircraft ${ }^{1}$ and, therefore, it is important to understand the noise sources associated with such configurations. To address this issue, a partnership effort between NASA and Gulfstream Aerospace Corporation was formed that is directed at understanding, and ultimately predicting the prominent sources of airframe noise. The main thrust of the NASA-Gulfstream partnership is targeted at acquiring a comprehensive aeroacoustic database on the principal sources of airframe noise. Availability of such a database is a crucial step toward improving our understanding of the pertinent noise generating mechanisms, and to develop noise reduction technologies. The focus of current work is on the Gulfstream nose landing gear configuration, which has been investigated both experimentally and computationally during the NASA-Gulfstream partnership over last several years.

Prediction of noise sources around the landing gear of an aircraft is an extremely challenging problem despite the progress made in the field of computational fluid dynamics (CFD) during the last several decades. The geometric complexity and highly chaotic unsteady flow fields associated with landing gear pose numerous difficulties for numerical simulation. For example, generating suitable point-matched structured grids for such configurations is an extremely difficult and time consuming task, making such an approach impractical. Hence, earlier structured grid work in this area focused on simplified landing gear configurations. ${ }^{2-4}$ The grid generation task for structured grids can be simplified by using an overset grid approach. However, such approaches in general do not maintain conservation properties across overset zones. It is suspected that the overset zones could introduce additional errors in acoustic waves propagating through the field.

Unstructured-grid methodology offers significant advantages in terms of grid generation and has been gaining popularity in recent years with an increasing emphasis on solving unsteady viscous flow over complex configurations, as evidenced by some recent examples appearing in the literature. ${ }^{5-11}$ For the current work, we make use of an unstructured-grid flow solver FUN3D, ${ }^{12}$ where the Navier-Stokes equations supplemented by turbulence models are solved to simulate the unsteady flow field. The FUN3D code has been used for many large-scale applications in the past including simulation of unsteady flow past single- and tandem- cylinder configurations, ${ }^{13}$ where time-averaged and perturbation quantities were shown to be in fairly good agreement with experimental data and earlier computations from the well-established, structured-grid flow solver CFL3D. ${ }^{14}$ Based on these earlier successes, the FUN3D code was selected for simulating the unsteady flow field associated with a representative nose landing gear configuration.

Prior work using the FUN3D code for landing gear simulations focused on the Gulfstream G550 nose landing gear installed in a closed-wall tunnel at NASA Langley Research Center, known as the Basic Aerodynamic Research Tunnel (BART). As reported by Vatsa et al., ${ }^{15}$ the resulting near-field solutions from these simulations were found to be in good agreement with measured data from the BART tunnel for surface pressures, and turbulence kinetic energy (TKE) and vorticity fields in wake regions of the gear. However, the accuracy of the predicted farfield noise was found to be less satisfactory, presumably because of the closed-wall tunnel configuration used in the simulations of Ref. 15 . In this paper, we focus on simulating the same landing gear installed in an open-jet tunnel, replicating the test set-up used for experimental measurements of the farfield noise, thus facilitating direct comparison of the predicted noise with measured data.

\section{Experimental Setup}

A series of wind tunnel experiments for a 1/4 scale model of a Gulfstream G550 aircraft nose landing gear have been performed at NASA Langley Research Center in BART, ${ }^{16}$ which is a closed-wall tunnel, and in the open-jet University of Florida Aeroacoustic Flow Facility (UFAFF). ${ }^{17}$ The BART facility is well suited for aerodynamic measurements including particle image velocimetry (PIV) measurements. However, due to the close proximity of tunnel walls, this facility is not suitable for noise data acquisition. In contrast, the UFAFF is an open-jet anechoic facility, 
designed for acquiring farfield noise measurements. The data from the BART facility is comprised of extensive steady and unsteady surface pressure and PIV measurements. Corresponding acoustic, and limited aerodynamic measurements, were acquired in the UFAFF facility to produce a comprehensive database. The wind tunnel experiments were conducted for both the fully-dressed Gulfstream G550 nose landing gear, as well as for the simplified, or partiallydressed, cavity-closed nose landing gear (PDCC-NLG) configuration. In addition to major components such as struts, cavity door, axle and wheels, the fully-dressed model also includes most of the smaller components, such as hydraulic and electric lines typical of flight hardware. In the simplified PDCC-NLG configuration, several smaller components, such as hydraulic and electric lines, the lighting cluster, and the steering assembly, were removed from the fullydressed model. Additionally, the door cavity, where the landing gear is stowed during the cruise mode, was closed in the PDCC-NLG configuration to create a simpler configuration for testing and code validation purposes. The comprehensive database developed from the BART and UFAFF tests for the simplified G550 PDCC-NLG configuration is currently being used as a benchmark to validate CFD and computational aeroacoustic (CAA) methods. ${ }^{18}$ The G550 PDCC-NLG is also the configuration examined in this paper.

The BART tests were conducted at a Reynolds number of $7.3 \times 10^{4}$ based on the main strut (piston) diameter and a free-stream Mach number of 0.166 . The Reynolds number in the UFAFF for the same inflow Mach number was $6.6 \times 10^{4}$ due to higher ambient temperature in Florida. Transition strips were attached on the model along the length of the strut to produce a turbulent boundary layer prior to flow separation on the strut. No such transition treatment was deemed necessary for the wheels and other components, which were made of composite materials resulting in higher surface roughness. Detailed steady pressure data is available for the wheels, door, and fuselage. In addition, unsteady pressure data was collected at 10 strategically located Kulite ${ }^{\circledR}$ pressure transducers. Off-surface PIV measurements were taken in the BART experiments to examine the detailed flow characteristics near critical components. Both phased and linear microphone arrays were used in the UFAFF for collecting noise data away from the landing gear.

\section{Governing Equations and Flow Solver}

In the present work, the spatially second-order accurate CFD solver FUN3D is used to obtain numerical solutions of the unsteady Navier-Stokes equations. A hybrid approach is used where the Reynolds-averaged Navier-Stokes (RANS) equations are solved in viscous regions near solid walls where the grid is inadequate to resolve the small scale unsteady flow structures, and a large eddy simulation (LES) approach is used in the remainder of the computational domain. The details of the switch and blending between these regions and the subgrid scale model are dependent on the turbulence model used for simulations. ${ }^{19-21}$

FUN3D is based on an unstructured-grid flow solver developed originally by Anderson and Bonhaus. ${ }^{22}$ This code has gone through significant modifications over the years by a team of researchers at NASA Langley using modern software practices. ${ }^{23}$ The discrete form of the governing equations are solved either in a time-accurate manner with a constant time step at every grid point or with variable time stepping to accelerate convergence to a steady state. At each iteration step, a linear system of equations is relaxed in a red-black fashion with a point implicit procedure. ${ }^{24}$ FUN3D can accommodate multiple types of grid elements including tetrahedrons, prisms, pyramids and hexahedrons, which makes it compatible with many different grid generators. For the current work, Roe's flux-difference splitting scheme $^{25}$ is used without a flux limiter.

A dual time-stepping algorithm with subiterations is used in FUN3D to converge the solution within each physical time-step. For these simulations, 15 subiterations per time-step were used, which was adequate to obtain at least three orders of magnitude reduction in the residuals of the governing equations. A variety of time marching schemes are available in FUN3D, including a second-order backward-differencing formulation (BDF2), and an optimized second order backward differencing formulation (BDF2OPT). The BDF2OPT scheme, ${ }^{26}$ which produces lower truncation error compared to the standard BDF2 scheme at nominally the same computational cost but with slightly increased memory usage, was chosen for the current application.

The Reynolds-averaged Navier-Stokes (RANS) equations are supplemented by turbulence model equations to simulate the effect of turbulence. Standard RANS turbulence models, such as the Spalart-Allmaras mode ${ }^{27}$ and the shear stress transport (SST) model of Menter, ${ }^{28}$ produce overly diffusive unsteady solutions in the presence of large separated flows encountered in the wake regions of bluff bodies, such as cylinders. ${ }^{29}$ The hybrid RANS/LES models such as the one-equation based Detached Eddy Simulation (DES) model of Spalart ${ }^{30}$ and the two-equation based HRLES model of Sanchez-Rocha et al. ${ }^{19}$ and Lynch and $\mathrm{Smith}^{20}$ have been shown to accurately capture the turbulent structures observed in wake and reverse flow regions for a variety of configurations. ${ }^{7,13}$ The hybrid RANS/LES

turbulence model under consideration here is based on the one-equation DES model of Spalart. ${ }^{30}$ Although the DES model has been used often for solving unsteady separated flows, this model depends too strongly on the grid quality 
and topology, and can lead to non-physical results with grid refinement in viscous layers. Spalart et al. ${ }^{31}$ have subsequently modified the DES model to overcome some of the shortcomings related to its grid dependence in a new model named Delayed Detached Eddy Simulation or DDES. The DDES model applies a blending function that varies between 0 for RANS mode and 1 for LES mode to the destruction terms. A slight modification of this model was suggested by Vatsa and Lockard ${ }^{13}$ to overcome numerical difficulties associated with the non-physical behavior of the eddy-viscosity in the upstream region of cylindrical bodies. In this model, the blending function suggested by Spalart et al. ${ }^{31}$ for the DDES model for the destruction terms is applied to the production terms also, and it will be referred here as the modified DDES, or "MDDES" model.

\section{Configuration and Grids}

The configuration under consideration here is the 1/4-scale, high-fidelity replica of a partially-dressed Gulfstream G550 nose landing gear that was tested by Zawodny et al. ${ }^{17}$ in the open-jet UFAFF tunnel. In a previous paper, Vatsa et al. ${ }^{15}$ examined the same configuration installed in the BART at NASA Langley. ${ }^{16} \mathrm{~A}$ set of three successively finer unstructured tetrahedral grids comprised of approximately 9,25 , and 71 million nodes $(54,150$, and 420 million cells) were generated using the VGRID grid generation software ${ }^{32}$ for the G550 PDCC-NLG configuration including the tunnel walls. For each grid refinement level, the average cell size was reduced by a factor of $\sqrt{2}$ in each coordinate direction. Grid spacing near the walls, and the stretching factors in the boundary-layer region were adjusted to yield nearly uniform grid refinement for this sequence of grids. Special attention was paid to grid resolution in the wake and shear-layer regions emanating from the door, cylindrical struts, and wheels of the gear assembly to resolve the associated unsteady flow structures. As reported in Ref. 15, the agreement of FUN3D solutions with the experimental data improved with grid refinement. In addition, significant improvement in numerical accuracy of the FUN3D solutions was attained at each grid level, when the tetrahedral grids were combined into mixed element grids consisting of prismatic elements in the high gradient boundary layer regions. The time-averaged surface pressures on wheels and cavity door obtained from the 71 million node ( 420 million cell) mixed-element grid FUN3D simulations compared well with the measured data. The agreement of computed results with the measured power spectral density (PSD) data was also quite good, except at the higher frequencies. The solutions obtained with the MDDES and HRLES turbulence models were nearly identical to each other.

A schematic of the computational domain chosen for current simulations of the nose landing gear configuration in the UFAFF is shown in Fig. 1, where the computational boundaries are displayed. In these simulations, the nose gear assembly is attached to a flat mounting plate, which is suspended in space. The computational domain enclosing the gear assembly and mounting plate is comprised of inflow, outflow, top, bottom and side boundaries shown in Fig. 1. A more detailed view of the isolated landing gear is shown in Fig. 2, where identifying numbers for the unsteady Kulite ${ }^{\circledR}$ pressure transducers are indicated in parenthesis next to the components of the landing gear.

Based on the experience gained during the simulation of the G550 nose landing gear assembly in the BART (Ref. 15), a grid density comparable to the finest grid (71 million nodes) used in that work was created for the surface and near vicinity of the landing gear. Additionally, this grid was enriched in a conical region surrounding the gear assembly and enclosing the flyover microphones that are directly under the gear, to facilitate comparison between acoustic propagation methodologies and the more direct CFD based noise computation. A planar cross-section of the grid region intersecting the mid-section of the landing gear is shown in Fig. 3, where the enriched grid in a conical region emanating from the gear and surrounding microphones (M4....M10) is clearly visible. The resulting unstructured grid generated with the VGRID software consists of approximately 146 million nodes (876 million cells).

\section{Results}

Although the Reynolds number based on a free-stream Mach number of 0.166 and the main strut (piston) diameter in UFAFF tests is $6.6 \times 10^{4}$, the computations here were performed at a Reynolds number of $7.3 \times 10^{4}$ to match the test conditions of the experimental study of Neuhart et al. ${ }^{16}$ in the BART at NASA Langley. This was done to provide a direct comparison of computational results between the BART and UFAFF configurations run with the same input conditions. Also, except for the noise data, most of the aerodynamic data available for comparisons was acquired in the BART facility. The computations were run in a fully-turbulent mode to replicate the experimental set up in which transition strips were used as needed to ensure turbulent separation. A constant wall temperature based on flat-plate adiabatic wall conditions and no-slip conditions were imposed on viscous surfaces, which included the gear, fuselage and mounting plate. At the inflow plane, total pressure, and total temperature corresponding to BART test conditions were imposed. Farfield conditions based on Riemann invariants were imposed at the other computational boundaries. 
The unstructured grids created by the VGRID code consist of purely tetrahedral cells (elements). However, VGRID uses an advancing layer technique to fill the viscous region adjacent to solid walls with semi-structured tetrahedral grids. A FUN3D pre-processing utility was used to combine these viscous region tetrahedrons back into prismatic elements to create mixed-element grids. Vatsa et al. ${ }^{15}$ demonstrated that mixed-element grids produced more accurate solutions compared to the solutions obtained on pure tetrahedral grids with the same number of nodes for the G550 PDCC-NLG configuration with respect to BART test data. It was also observed from these earlier studies, that the results obtained with the one-equation MDDES turbulence model compared well with the experimental data, and were very similar to the results obtained with the two-equation HRLES model. Therefore, the current simulations were performed using the MDDES model on mixed-element grids for efficiency reasons.

The FUN3D code was first run in steady mode starting from free-stream conditions to expedite the development of the mean flow. This was followed by 20,000 time steps run in time-accurate mode using a time-step of $4.92 \times 10^{-6}$, which advanced the solutions by a physical time of 0.0984 secs. Based on the free-stream convective speed, the disturbances travel a distance of approximately 40 wheel diameters or twice the length of the BART tunnel during this time, which is considered adequate to purge the initial disturbances out of the computational domain. Temporalaveraging was activated during the next 20,000 time-steps to establish a well-converged baseline mean flowfield before collecting the unsteady data for acoustic analysis. Finally, another 70,000 time steps were run to provide sufficient data for acoustic analysis. In these simulations, the unsteady data was collected on solid surfaces and on a permeable surface enclosing the gear assembly, the fuselage, and the mounting plate for farfield noise prediction using the Ffowcs Williams-Hawkings (FW-H) approach. ${ }^{33}$ In addition, the pressure perturbation data at the microphone locations (see Fig. 3) was also collected for direct comparison with the experimental data.

Care was taken to ascertain that the numerical solutions were converged well within each time-step sub-iteration loop. The physical time-step for these computations was chosen so that 20 time steps per period were used for a 10 $\mathrm{KHz}$ signal, which represents the highest frequency of interest for this work because noise levels beyond this frequency are not significant. The number of time steps per period is inversely proportional to the frequency, and therefore the temporal resolution at lower frequencies is much higher.

The time-averaged surface pressures obtained using the MDDES model are compared with experimentally measured data in UFAFF for the starboard wheel of the landing gear in Fig. 4. The computational results are in very good agreement with the measured surface pressure data for the wheel. It is observed from this figure that the computed pressure distributions accurately capture the pressure levels and shape of the curves, including the peaks and troughs.

Next we examine the pressure distributions on the nose-gear door. Experimental measurements were taken along 9 rows of ports mounted on the door surface as shown in Fig. 5. The computed pressures along rows $2-4$, and rows 5-7 are compared with the measured data in Figs. 6 (a) and 6(b), respectively. Because of the sparseness of the experimental data, it is difficult to ascertain the precise shape of the experimental curves and to draw firm conclusions about these comparisons. In general, the computed pressures compare reasonably well with the measurements in the middle portion of the door, the largest disagreement being for row 3 . There is a steep drop in computed pressure levels towards the door edges due to sudden acceleration of the flow at the door edges, that is not captured in the experimental data due to the lack of instrumentation in these regions.

The computed results from the current simulations for the 2-D turbulence kinetic energy (2-D TKE), $\left[1 / 2\left(u^{\prime 2}+\right.\right.$ $\left.v^{\prime 2}\right)$ ], for a plane downstream of the mid-section of the wheels, denoted as wheel_plane3 in Fig. 7, are compared in Fig. 8 with the PIV data obtained from the experiments conducted in BART. ${ }^{16}$ No such data is available from UFAFF tests at this time. Although the 2-D TKE levels are overpredicted, the computed results are qualitatively similar to the measured data, and display the correct shape and trends for the decay of TKE levels in the wheel wake and shear layer regions. The computational results shown here are very similar to the finest grid results presented in Ref. 15 for the BART configuration.

Next, we examine the surface pressure power spectral density (PSD) distributions at different locations on the gear corresponding to the position of Kulite ${ }^{\circledR}$ pressure transducers, which are depicted in Fig. 2 for reference. The computational solutions from this study are compared with the experimental data from the UFAFF ${ }^{17}$ in Fig. 9 at two locations on the door corresponding to Kulite ${ }^{\circledR}$ pressure transducers (channels) 3 and 10, and in Fig. 10 at transducers 7 (wheel) and 15 (lower torque-arm). The computational results from the finest grid of Ref. 15 simulating the BART configuration are also plotted in these figures. Based on these comparisons, the computed PSD distributions at the surface-mounted Kulite ${ }^{\circledR}$ locations display only small differences for these two configurations, where the computed PSD levels for the BART configuration appear slightly higher compared to the UFAFF simulation in the low- to midfrequency range. In addition, the overall agreement between the simulated and measured data is quite good up to a frequency of $3 \mathrm{KHz}$. 


\section{A. Farfield Noise}

Zawodny et al. ${ }^{17}$ have conducted aeroacoustic testing in UFAFF, an open jet anechoic tunnel of the same nose landing gear model that was tested in the closed-wall NASA Langley's BART tunnel. In this experiment, a series of 11 microphones were placed at a distance of several wheel diameters underneath the nose gear along the centerline of the gear as shown in Fig. 11. The spectra at the microphone locations were corrected to account for shear layer refraction. Note that the effective position of microphone 7 shown in Fig. 11 is directly underneath the nose gear, which closely represents an overhead flyover position of $90^{\circ}$.

In this paper, we have computed the farfield noise with three different approaches. In the first approach, the noise levels were computed directly from the unsteady CFD data collected at selected points in the field. The second approach is the more conventional hybrid CFD/Acoustic Analogy Approach, where the pressure fluctuations on the solid surfaces of the landing gear configuration obtained from the CFD solutions were used as input to the Ffowcs Williams-Hawkings ${ }^{33}$ (FW-H) solver PSU-WOPWOP ${ }^{34,35}$ to predict the farfield noise. The third and final approach makes use of FW-H solver to propagate the CFD generated unsteady flowfield fluctuations (acoustic signal) from a permeable surface enclosing both the surface and volumetric noise sources to points of interest in the farfield.

\section{Direct prediction of noise}

For the direct prediction of noise, the unsteady data collected at selected points in the computational domain (denoted as black circles in Fig. 3) is processed through a fast Fourier transform (FFT) and converted to power spectral density (PSD) and 1/3 octave sound pressure levels (SPL). The noise data obtained in this manner is therefore extracted directly from the CFD solutions without relying on acoustic propagation techniques. This approach was used initially to extract the PSD levels at a series of points along a vertical line underneath the gear denoted as R1-R9 on Fig. 3. The computed PSD levels at the points R1-R8 normalized by $1 / r$ are shown in Fig. 12, where $r$ is the distance of these points from the mounting plate. Point R9, which clearly resides outside the enriched mesh region is excluded from this figure. These results indicate that the PSD levels for points R3-R7 collapse very nicely up to a frequency of approximately $7 \mathrm{KHz}$ with $1 / r$ normalization, displaying a typical farfield decay of acoustic signal. Note that the results for points R1 and R2, which are situated closer to the wheels, lie slightly above the collapsed data. The offset from the collapsed data is smaller for point R2 which is physically located further away from the wheels as compared to point R1. The decrease in PSD levels for point R8 away from the other points at frequencies above $2.5 \mathrm{KHz}$ is attributed to the coarseness of the grid surrounding this point. The present results clearly demonstrate that accurate prediction of noise levels directly from CFD solutions is achievable, but it comes at a significant cost because such an approach requires a high-density mesh not only in the boundary layer, shear layer, and wake regions associated with large hydrodynamic gradients, but even in the farfield where the hydrodynamic field is relatively invariant. This was one of the primary reasons for using a high density mesh all the way up to the flyover microphones (M4-M10) in these simulations, as seen in Fig. 3. The noise levels computed using the direct approach at selected microphones will be compared with the measured data and other numerical results later in this section.

\section{Noise prediction using solid surface data}

In this conventional aeroacoustic analogy approach, the perturbation pressure data collected on all the solid surfaces including the nose landing gear assembly, fuselage, and tunnel floor (or mounting plate in UFAFF) are used in various combinations as input to the FW-H solver PSU-WOPWOP ${ }^{34,35}$ for computing the $1 / 3$ Octave SPL levels in $\mathrm{dB}$ at farfield points. The results obtained in this manner at flyover microphones 4, 7, and 9 are shown in Figs. $13-15$, based on the current UFAFF simulations and the 71 million node grid BART simulation of Ref. 15. In these figures, we have plotted the computational results obtained by using the unsteady pressure contributions from all the pertinent solid surfaces, i.e. the gear, fuselage and tunnel floor (mounting plate in UFAFF) as solid red lines. The experimental data from the UFAFF, which represents the noise levels at these locations from all the sources for this configuration, is shown as solid black lines connecting filled circles. It is clear from these figures that the results based on the openjet UFAFF simulations are in much better agreement with the measured data compared to results from simulation of closed-wall BART configuration.

To quantify the effect of individual components on noise levels, we also show the results when the contributions from the floor, and subsequently, the floor and the fuselage are excluded from the noise computation. By examining the results presented in this manner in Figs. 13 - 15, it is clear that the contributions from the tunnel floor and the fuselage dominate the farfield noise at these microphone locations for the BART simulations. By contrast, the primary contribution to the farfield noise in UFAFF simulations comes from the gear itself. Nonetheless, the contributions 
from the fuselage and mounting plate are non-negligible in the overhead and downstream directions, especially at low frequencies. Although there is some interaction between vortical structures and the fuselage, the fuselage and mounting plate also act as reflective surfaces for sound waves emanating from the gear. In Figs. 14 and 15, the total signal around $700 \mathrm{~Hz}$ is actually lower than that from the Gear + Fuselage, indicating that the phasing of the reflections is important, causing both constructive and destructive interference. It is conjectured that since BART is a closed-wall facility, the reflections from the tunnel walls are trapped and excite duct modes which the FW-H solver (based on a free-space Green's function) detects but cannot interpret correctly. As more surface area subject to the trapped acoustic reflections is included, the predicted noise levels increase. By comparing the Gear-alone results from the UFAFF and BART configurations in Figs. 13 - 15, one can see that although the general shapes are similar, the BART results are several $\mathrm{dB}$ higher. Hence, even the gear alone result is likely influenced by the internal reflections in the BART case. In general, the predicted SPL levels obtained by using the surface pressure obtained from CFD simulations for the UFAFF configuration in conjunction with FW-H propagation code are in much better agreement with the measured data. Also, the Gear + Floor + Fuselage result, which best simulates the experimental configuration, is closer to the experimental data than the Gear-alone prediction. However, one could probably assess the general character of the noise without including the mounting plate.

\section{3. $\quad$ Noise prediction using permeable surface data}

An alternative, and sometimes preferred approach for computing farfield noise, is to use the unsteady flowfield data on a virtual permeable (porous) surface enclosing the surface and volumetric noise sources in conjunction with FW$\mathrm{H}$ noise propagating solvers. The permeable surface, which serves as a control volume, should preferably reside outside of strongly varying hydrodynamic regions but still within the computational domain where good resolution is maintained. Perspective and front views of the permeable surface used in these simulations are shown in Figs. 16 (a) and (b). In these figures, the gear assembly is shown in red color and the mounting plate is colored blue. The permeable surface, which is colored yellow, encloses the gear assembly, fuselage, and the mounting plate. The permeable surface also extends approximately 12 wheel diameters downstream of the gear, thus enclosing the most energetic portion of flow associated with the wake behind the nose gear assembly.

The unsteady fluctuations obtained from the FUN3D code on the permeable surface shown in Fig. 16 were used as input to the FW-H solver PSU-WOPWOP for predicting the farfield noise. The resulting noise levels obtained in this manner are compared with the experimental data and the predictions made using the pressure perturbations on solid surfaces in Figs. 17 (a)-(c). The overall agreement between the predicted noise using unsteady pressure data on solid surfaces and flow-data on permeable surface is quite good indicating a very weak contribution from the volumetric sources to the farfield noise levels. Based on these comparisons, it appears that for this type of configurations we can reliably make use of the solid surface perturbation data for noise prediction, which results in a simpler data collection procedure.

As mentioned earlier in this section, we also had collected the fluctuating unsteady pressure data corresponding to the microphone locations during the CFD simulation of the UFAFF configuration. This data was processed through a fast Fourier transform (FFT) and converted to $1 / 3$ octave sound pressure levels (SPL). The noise data obtained in this manner is therefore extracted directly from the CFD solutions without relying on acoustic propagation techniques. The computed SPL distributions labeled "FUN3D (Direct approach)" for microphones 4, 7, and 9 are compared with the measurements in Figs. 17 (a)-(c), respectively. In general, the overall agreement between the results obtained directly from FUN3D at these microphone locations and the FW-H solutions is quite good. The largest discrepancy between the results obtained with these two different computational approaches is seen for microphone 9, which is situated relatively close to the inflow plane and is likely influenced by reflections off the upstream boundary that have been observed in an animation of the CFD simulation data. We also notice a drop-off in SPL levels in the FUN3D results for microphone 4 at a frequency of $3 \mathrm{KHz}$. Compared to the other two microphones examined here, the grid density enclosing this microphone is somewhat coarser (see Fig. 3), which explains the loss of accuracy at the higher frequencies. Such strict grid requirements make the use of the direct approach less practical, especially for predicting noise levels far away from the noise producing components at large number of observer locations. In general, it is more economical to use the FW-H propagation codes in combination with the CFD solvers for farfield noise computation compared to the direct approach based solely on CFD solutions. 


\section{Concluding Remarks}

Numerical simulations have been performed for a partially-dressed cavity-closed nose landing gear configuration that was tested in the NASA Langley's closed-wall BART tunnel and the University of Florida's open-jet tunnel known as the UFAFF. In the current work, the unstructured-grid flow solver FUN3D has been used to simulate the experimental set up of the UFAFF where the noise measurements were performed. The FUN3D solver was run in a time-accurate mode with the MDDES turbulence model, which is a hybrid RANS/LES model, to capture the noiseproducing unsteady flow structures. Making use of the unsteady data acquired during these simulations, the power spectral density and $1 / 3$ octave sound pressure levels have been computed. The sound pressure levels computed using the unsteady data from solid surfaces and from a permeable surface surrounding the gear assembly are found to be in good agreement with each other and with the measured data. Compared to earlier results obtained from simulation of the same configuration in the BART tunnel, significant improvement is shown for the prediction of farfield sound pressure levels.

It was demonstrated in this paper that the noise levels in the farfield can also be computed directly from the unsteady flow field data generated during the CFD simulations. However, such an approach requires high density grids even in the farfield, making such an approach less efficient for practical applications.

One of the major difficulties faced in such simulations is the generation of grids that are suitable for resolving the near wall viscous regions as well as the shear layers and wake regions away from solid surfaces. In general, along with a priori knowledge of the flow field, a lot of experience and trial and error are required to manually generate suitable grids for such applications. For this reason, our future work would focus on making use of the grid adaption capability of the FUN3D code to improve the overall computational efficiency to perform such calculations.

\section{Acknowledgments}

This work was supported by NASA's Fundamental Aerodynamics and Integrated Systems Research Programs through the Subsonic Fixed-Wing and Environmentally Responsible Aviation projects. The authors would also like to acknowledge Mr. Michael Wiese of Vigyan, Inc. for generating the grids used in this work.

\section{References}

\footnotetext{
${ }^{1}$ Khorrami, M. R., Lockard, D. P., Humphreys, Jr., W. M., Choudhari, M. M., and Van de Ven, T., "Preliminary Analysis of Acoustic Measurements from the NASA-Gulfstream Airframe Noise Flight Test," AIAA Paper 2008-2814, May 2008.

${ }^{2}$ Lockard, D. P., Khorrami, M. R., and Li, F., "High Resolution Calculation of a Simplified Landing Gear," AIAA Paper 2004-2887, May 2004.

${ }^{3}$ Spalart, S., Shur, M., Strelets, M., and Travin, A., "Towards Noise Prediction for Rudimentary Landing Gear," IUTAM Symposium on Computational Aero-Acoustics for Aircraft Noise Prediction: ScienceDirect, Vol. 1, pp. 283-292, 2010.

${ }^{4}$ Li, F., Khorrami, M., and Malik, M., "Unsteady Simulation of a Landing-Gear Flow Field,” AIAA Paper 2002-2411, June 2002.

${ }^{5}$ Morton, S., McDaniel, D., and Cummings, R., "F-16XL Unsteady Simulations for the CAWAPI Facet of RTO Task Group AVT-113," AIAA Paper 2007-0493, January 2007.

${ }^{6}$ Morton, S., Cummings, R., and Kholodar, D., "High Resolution Turbulence Treatment of F/A-18 Tail Buffet," Journal of Aircraft, Vol. 44, No. 6, 2007, pp. 1769-1775.

${ }^{7}$ Cummings, R., Morton, S., and McDaniel, D., "Experiences in Accurately Predicting Time-dependent Flows," Progress in Aerospace Sciences, Vol. 44, No. 4, 2008, pp. 241-257.

${ }^{8}$ Biedron, R. and Lee-Rausch, E., "Rotor Airloads Prediction using Unstructured Meshes and Loose CFD/CSD Coupling," AIAA Paper 2008-7341, August 2008.

${ }^{9}$ Bartels, R., Chwalowski, P., Massey, S., Heeg, J., Wiesemann, C., and Mineck, R., "Computational Aeroelastic Analysis of the Ares Launch Vehicle During Ascent,” AIAA Paper 2010-4374, June 2010.

${ }^{10}$ Forsythe, J., Strang, W., and Squires, K., "Six Degree of Freedom Computation of the F-15E Entering a Spin," AIAA Paper 2006-858, January 2006.

${ }^{11}$ Cameli, F., Lohner, R., and Hanna, S., "VLES Study of Flow and Dispersion Patterns in Heterogeneous Urban Areas," AIAA Paper 20061419, January 2006.

${ }^{12}$ FUN3D Web page: http://fun3d.larc.nasa.gov, November 2011.

${ }^{13}$ Vatsa, V. and Lockard, D., "Assessment of Hybrid RANS/LES Turbulence Models for Aeroacoustics Applications," AIAA Paper 2010-4001, June 2010.

${ }^{14}$ Rumsey, C., Biedron, R., and Thomas, J., “CFL3D: Its History and Some Recent Applications,” NASA TM 112861, May 1997.

${ }^{15}$ Vatsa, V., Lockard, D., and Khorrami, M., "Application of FUN3D Solver for Aeroacoustics Simulation of a Nose Landing Gear Configuration," AIAA Paper 2011-2820, June 2011.

${ }^{16}$ Neuhart, D., Khorrami, M., and Choudhari, M., “Aerodynamics of a Gulfstream G550 Nose Landing Gear Model,” AIAA Paper 2009-3152, May 2009.
} 
${ }^{17}$ Zawodny, N., Liu, F., Yardibi, T., Cattafesta, L., Khorrami, M., Neuhart, D., and Van de Ven, T., "A Comparative Study of a 1/4-scale Gulfstream G550 Aircraft Nose Gear Model," AIAA Paper 2009-3153, May 2009.

${ }^{18}$ BANCII Web page: https://info.aiaa.org/tac/ASG/FDTC/DG/BECAN.aspx, May 2012.

${ }^{19}$ Sanchez-Rocha, M., Kirtas, M., and Menon, S., "Zonal Hybrid RANS-LES Method for Static and Oscillating Airfoils and Wings," AIAA Paper 2006-1256, January 2006.

${ }^{20}$ Lynch, C. and Smith, M. J., "Hybrid RANS-LES Turbulence Models on Unstructured Grids," AIAA Paper 2008-3854, June 2008.

${ }^{21}$ Baurle, R. and Edwards, J., "Hybrid Reynolds-Averaged/Large-Eddy Simulations of a Coaxial Supersonic Free-Jet Experiment," AIAA Paper 2009-0129, January 2009.

${ }^{22}$ Anderson, W. K. and Bonhaus, D. L., “An Implicit Upwind Algorithm for Computing Turbulent Flows on Unstructured Grids," Computers and Fluids, Vol. 23, No. 1, 1994, pp. 1-21.

${ }^{23}$ Alexandrov, N., Atkins, H. L., Bibb, K. L., Biedron, R. T., Gnoffo, P. A., Hammond, D. P., Jones, W. T., Kleb, W. L., Lee-Rausch, E. M., Nielsen, E. J., Park, M. A., Raman, V. V., Roberts, T. W., Thomas, J. L., Vatsa, V. N., Viken, S. A., White, J. A., and Wood, W. A., "Team Software Development for Aerothermodynamic and Aerodynamic Analysis and Design,” NASA TM 2003-212421, Nov. 2003.

${ }^{24}$ Nielsen, E., Lu, J., Park, M., and Darmofal, D., "An Implicit, Exact Dual Adjoint Solution Method for Turbulent Flows on Unstructured Grids," Computers and Fluids, Vol. 33, No. 9, 2003, pp. 1131-1155.

${ }^{25}$ Roe, P. L., "Approximate Riemann Solvers, Parameter Vectors, and Difference Schemes," Journal of Computational Physics, Vol. 43, No. 2, 1981, pp. 357-372.

${ }^{26}$ Vatsa, V., Carpenter, M., and Lockard, D., "Re-evaluation of an Optimized Second Order Backward Difference (BDF2OPT) Scheme for Unsteady Flow Applications," AIAA Paper 2010-0122, January 2010.

${ }^{27}$ Spalart, P. R. and Allmaras, S., "A One-Equation Turbulence Model for Aerodynamic Flows," La Recherche Aerospatiale, Vol. 1, No. 1, 1994, pp. 5-21.

${ }^{28}$ Menter, F. R., "Two-equation Eddy-viscosity Turbulence Models for Engineering Applications," AIAA Journal, Vol. 32, No. 8, 1994, pp. $1598-1605$.

${ }^{29}$ Vatsa, V. N. and Singer, B., "Evaluation of a Second-Order Accurate Navier-Stokes Code for Detached Eddy Simulation Past a Circular Cylinder," AIAA Paper 2003-4085, June 2003.

${ }^{30}$ Spalart, P. R., "Young Person's Guide to Detached-Eddy Simulation Grids," NASA CR 211032, July 2001.

${ }^{31}$ Spalart, P. R., Deck, S., Shur, M. L., Squires, K. D., Strelets, M. K., and Travin, A., "A New Version of Detached-Eddy Simulation, Resistant to Ambiguous Grid Densities," Theoretical and Computational Fluid Dynamics, Vol. 20, No. 3, 2006, pp. 181-195.

${ }^{32}$ Pirzadeh, S., "Three-dimensional Unstructured Viscous Grids by the Advancing Layer Method," AIAA Journal, Vol. 33, No. 1, 1996, pp. 4349.

${ }^{33}$ Ffowcs Williams, J. E. and Hawkings, D. L., "Sound Generated by Turbulence and Surfaces in Arbitrary Motion," Philosophical Transactions of the Royal Society, Vol. A264, No. 1151, 1969, pp. 321-342.

${ }^{34}$ Brentner, K. S., Lopes, L. V., Chen, H. N., and Horn, J. F., "Near Real-Time Simulation of Rotorcraft Acoustics and Flight Dynamics," Proceedings of the American Helicopter Society 59th Annual Forum, 2003.

${ }^{35}$ Bres, G. A., Brentner, K. S., Perez, G., and Jones, H. E., "Maneuvering Rotorcraft Noise Prediction," Journal of Sound and Vibration, Vol. 275, No. 3-5, 2004, pp. 719-738. 


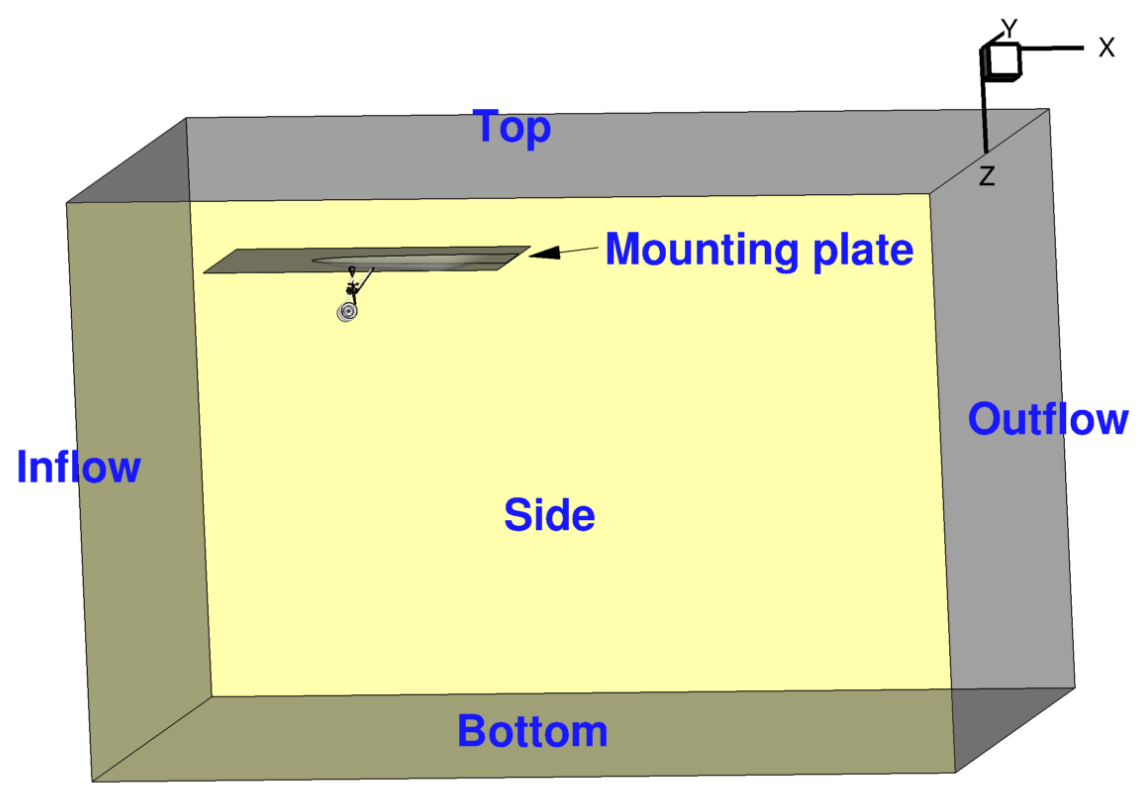

Figure 1. Computational model of the nose landing gear in UFAFF tunnel.

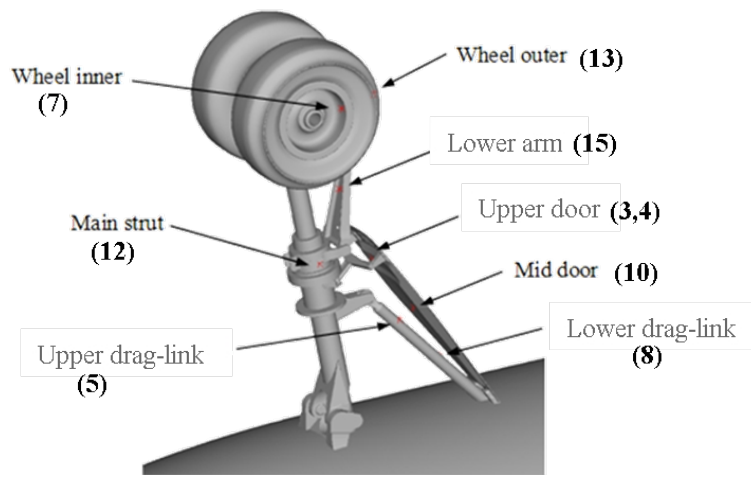

Figure 2. Kulite ${ }^{\mathbb{B}}$ pressure transducer locations and nomenclature for nose landing gear, transducer numbers are given in parentheses.

10 of 18

American Institute of Aeronautics and Astronautics Paper 2010-4001 


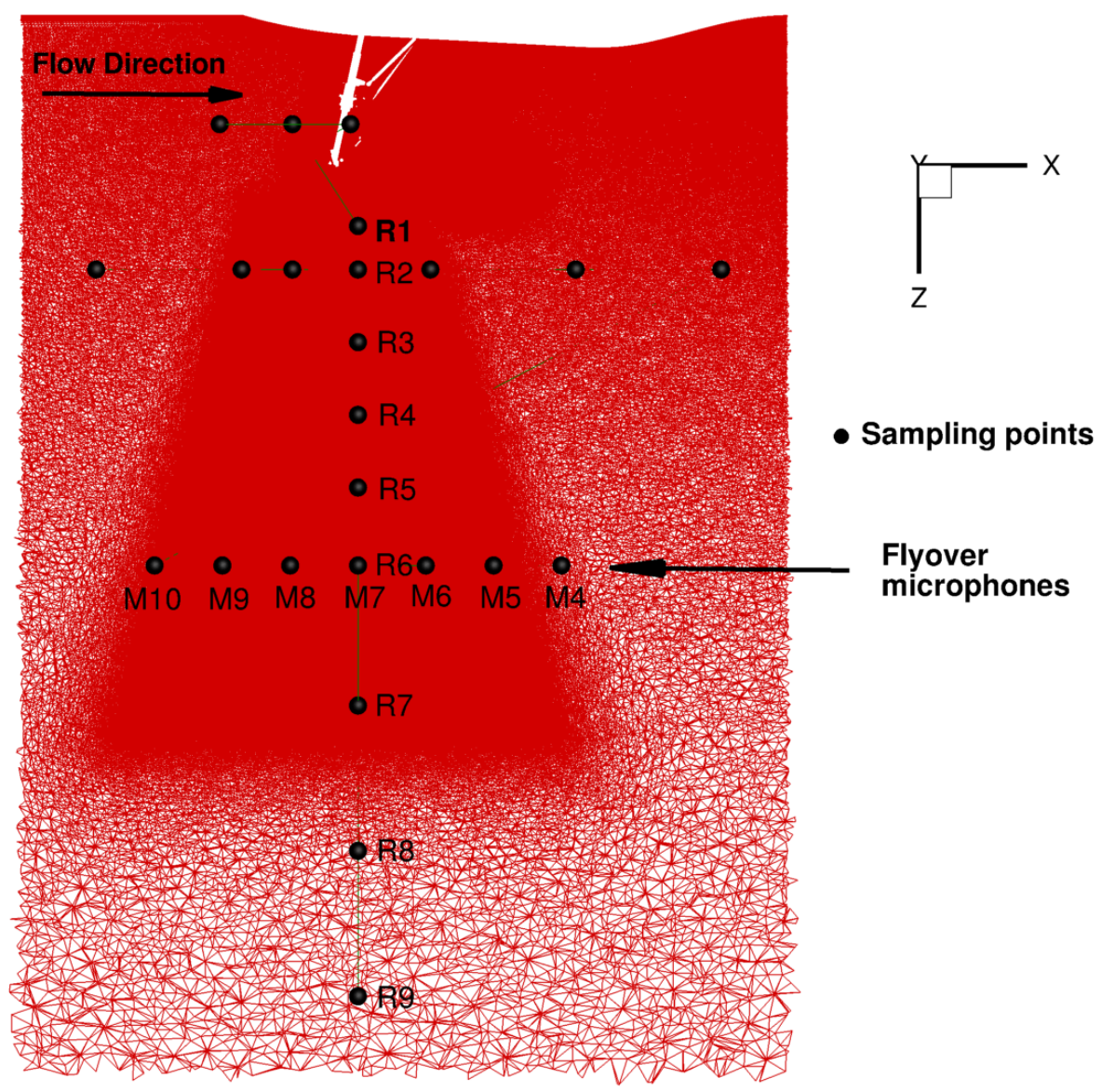

Figure 3. Sampling point locations for unsteady data collection, $y=0$ plane. 


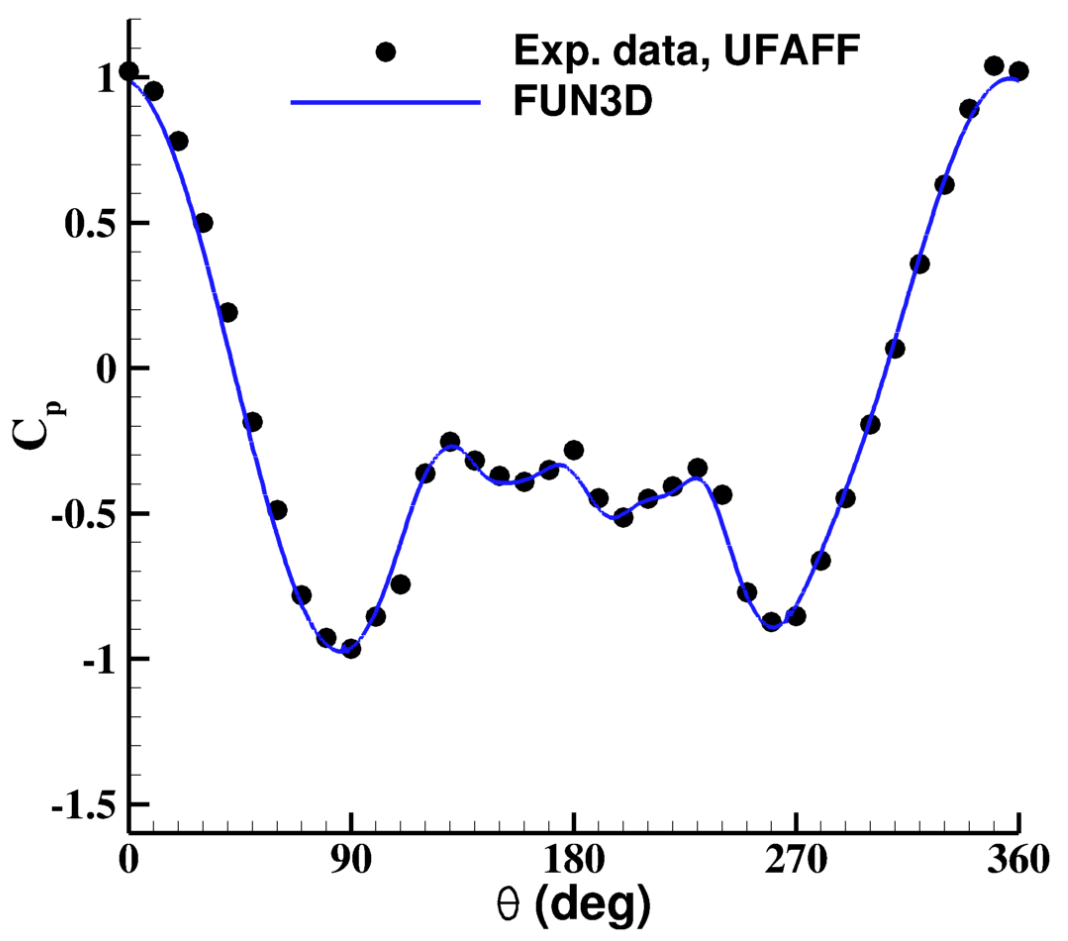

Figure 4. Surface pressure distributions on starboard wheel.

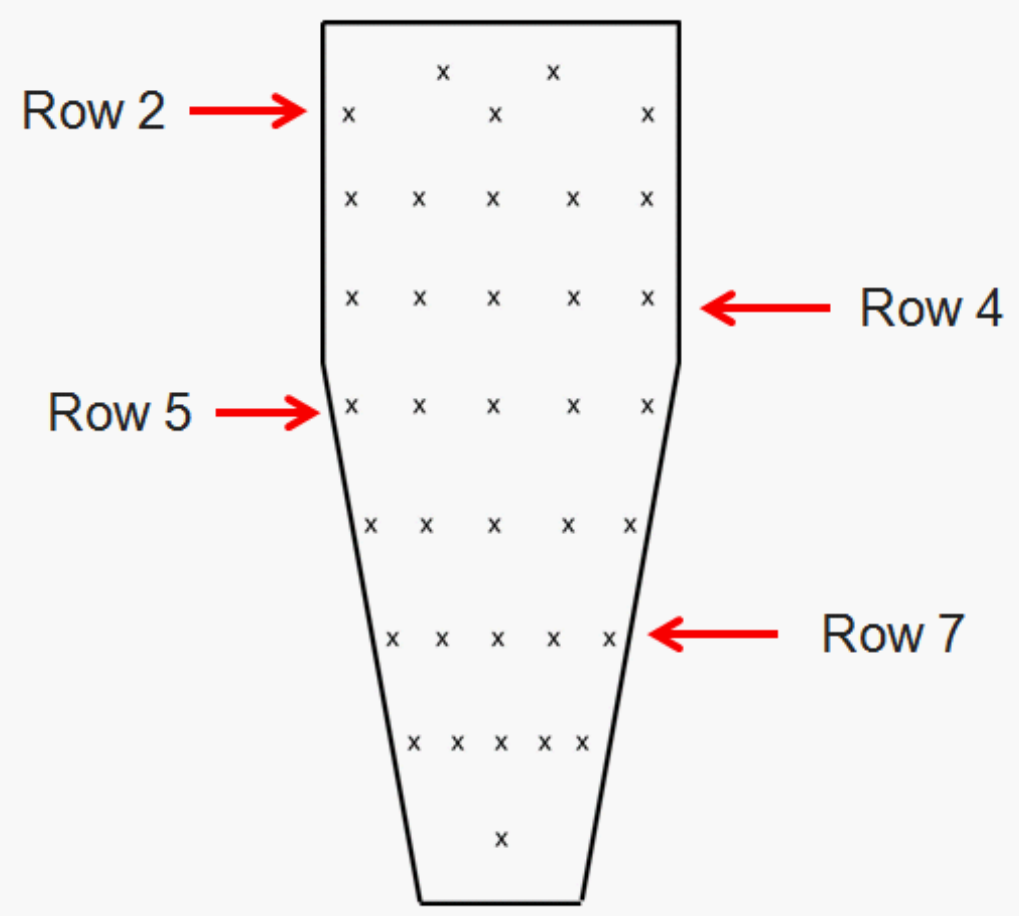

Figure 5. Nomenclature for rows on cavity door. 


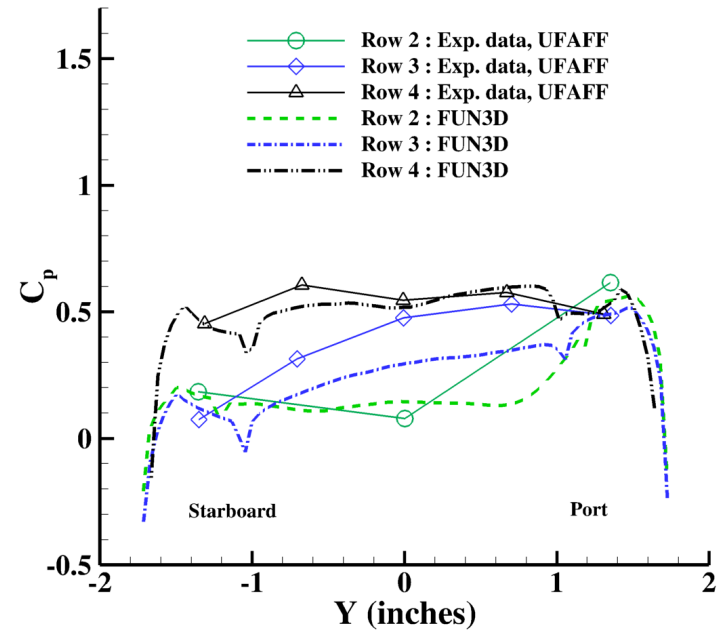

(a) Rows 2-4

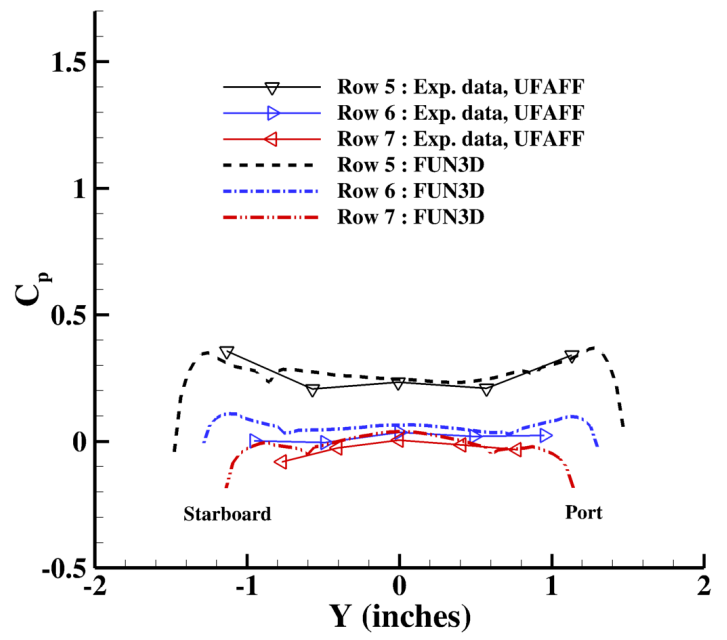

(b) Rows 5-7

Figure 6. Surface pressure distributions on nose-gear door.

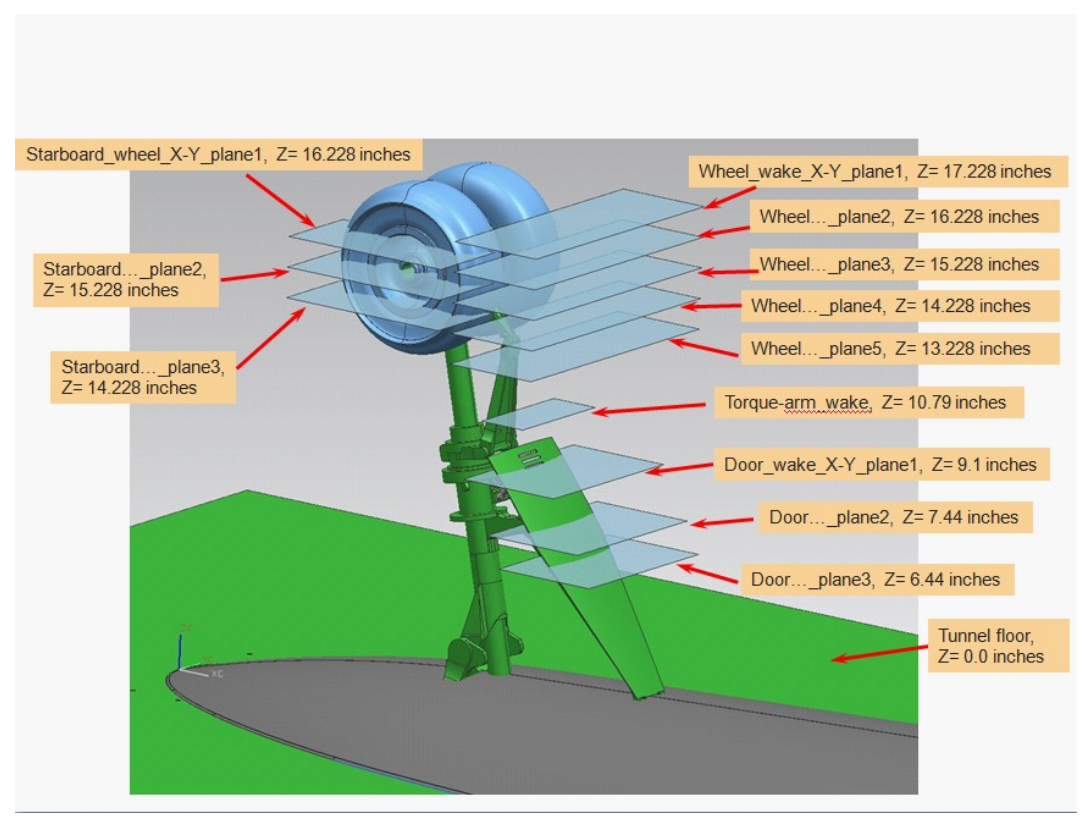

Figure 7. PIV measurement planes for BART experimental set up 


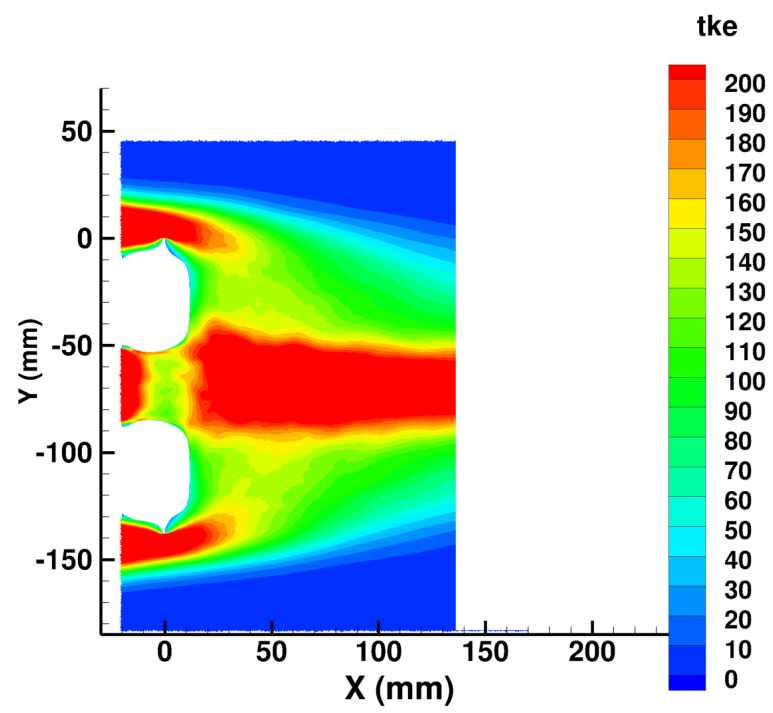

(a) FUN3D simulation

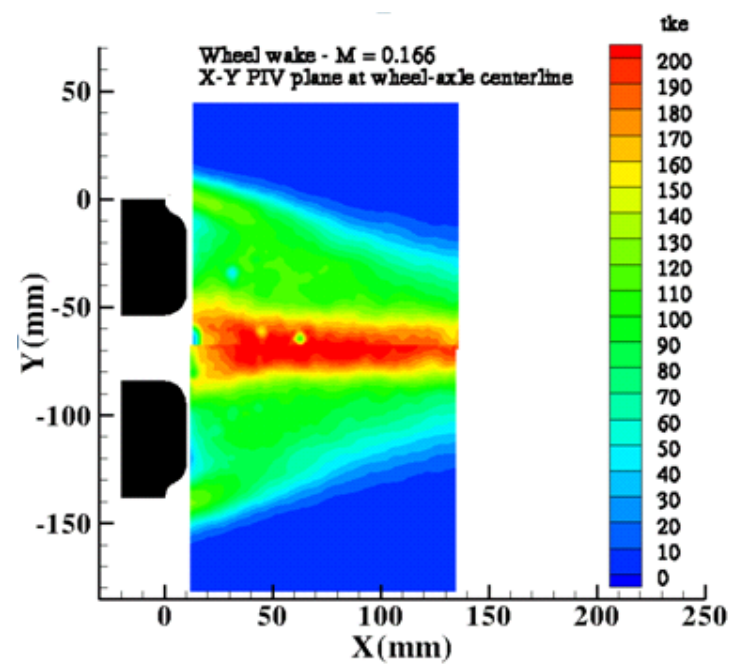

(b) Exp. PIV data

Figure 8. 2-D Turbulence kinetic energy contours, mid-wheel plane3.

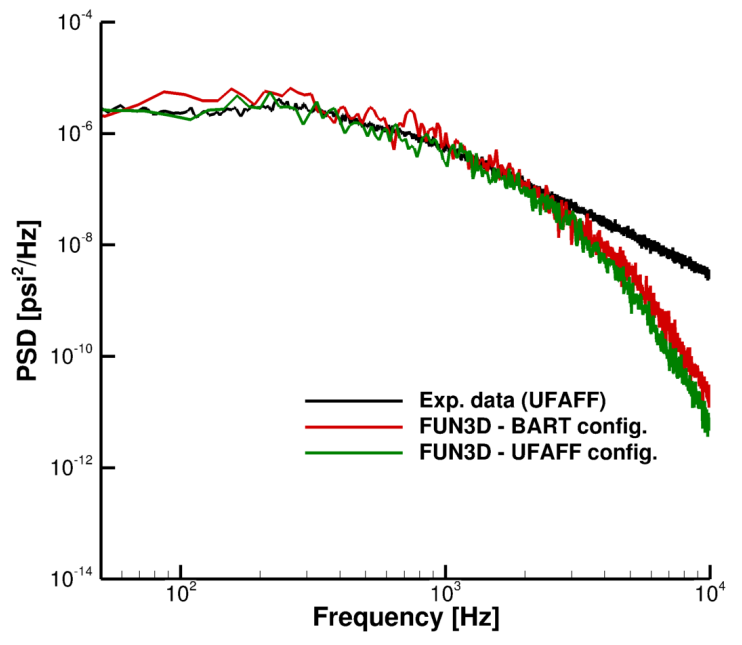

(a) Upper-door, ch. 3

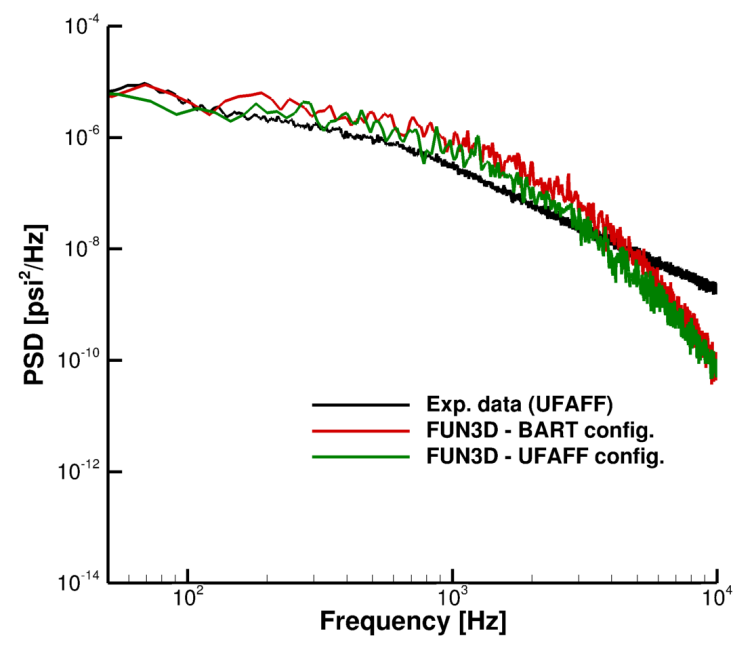

(b) Mid-door, ch. 10

Figure 9. PSD comparisons for Kulite ${ }^{\circledR}$ transducers 3 and 10. 


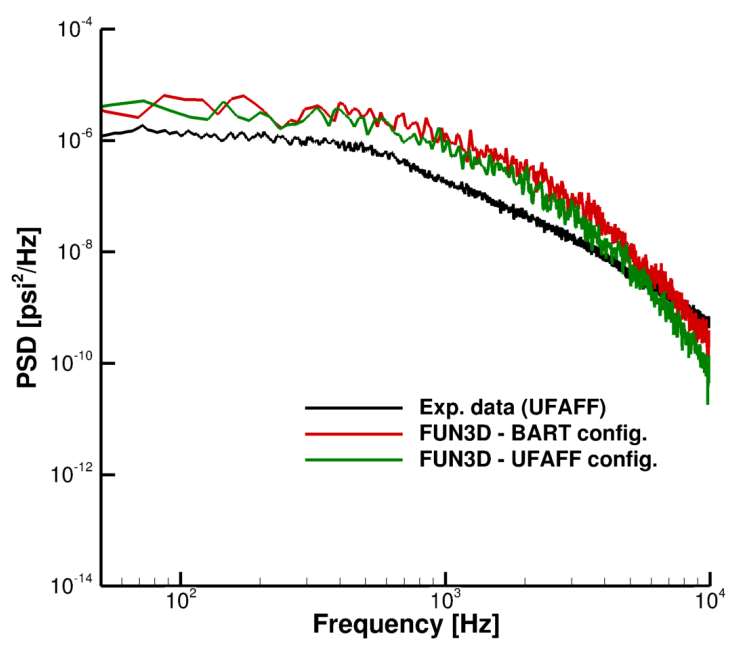

(a) Wheel-inner, ch. 7

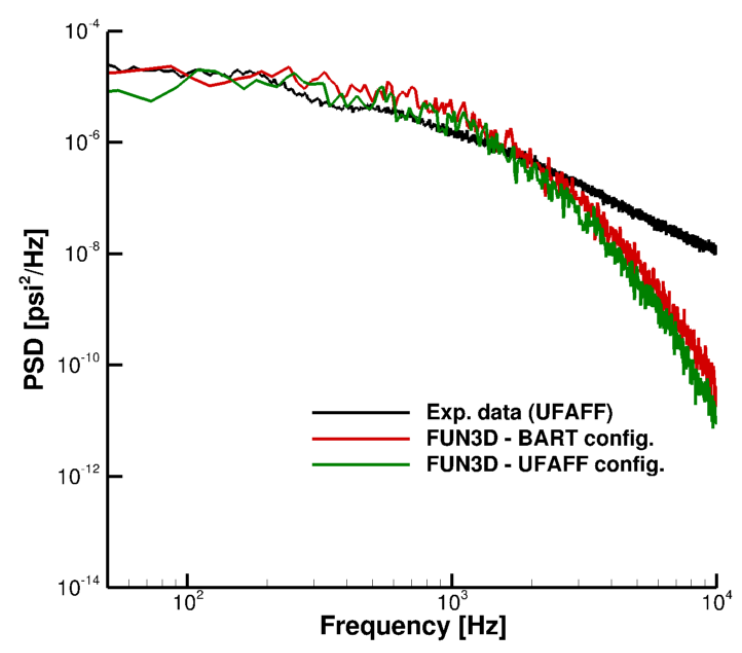

(b) Lower arm, ch. 15

Figure 10. PSD comparisons for Kulite ${ }^{\circledR}$ transducers 7 and 15.

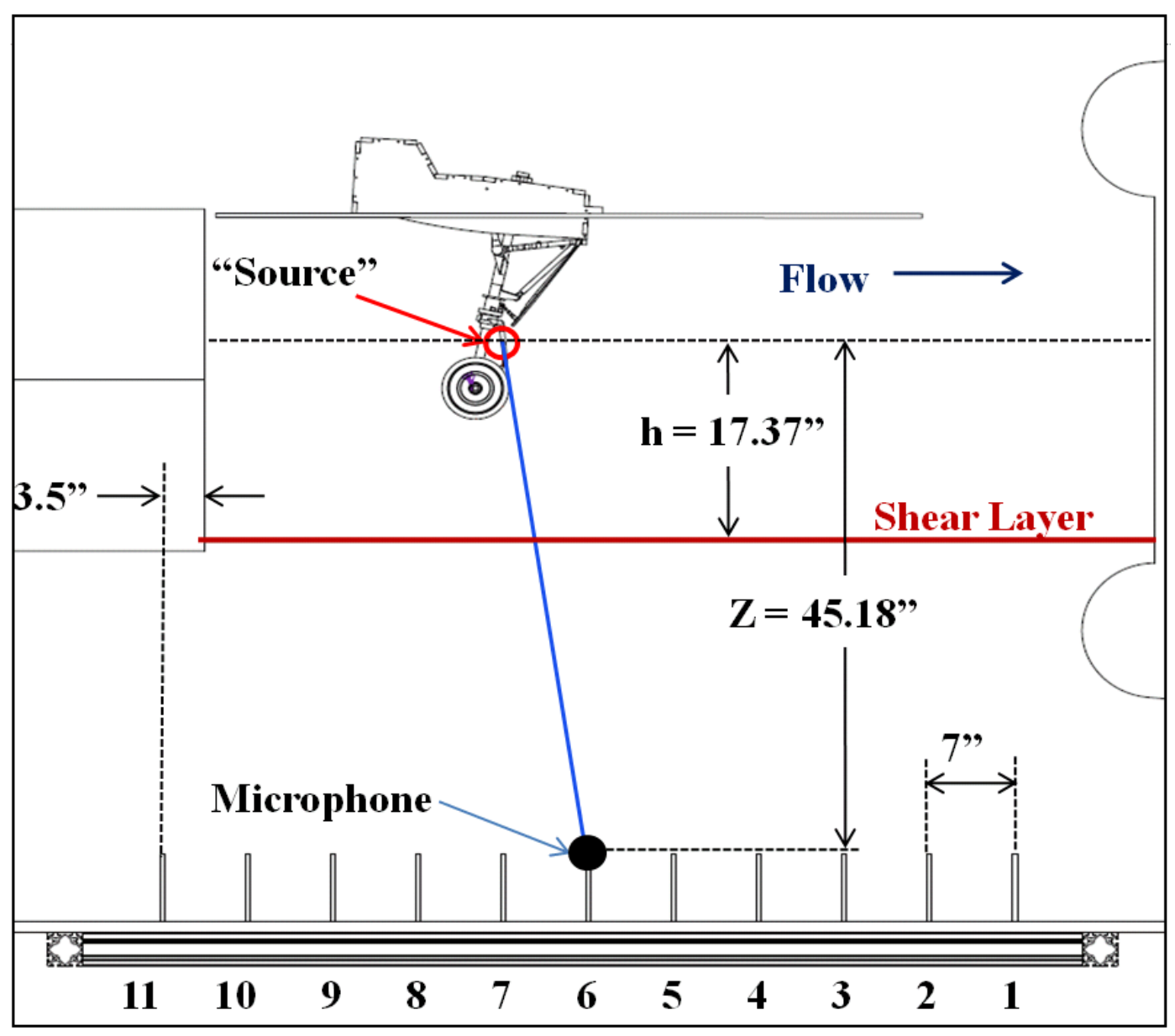

Figure 11. Linear microphone array arrangement in UFAFF. 


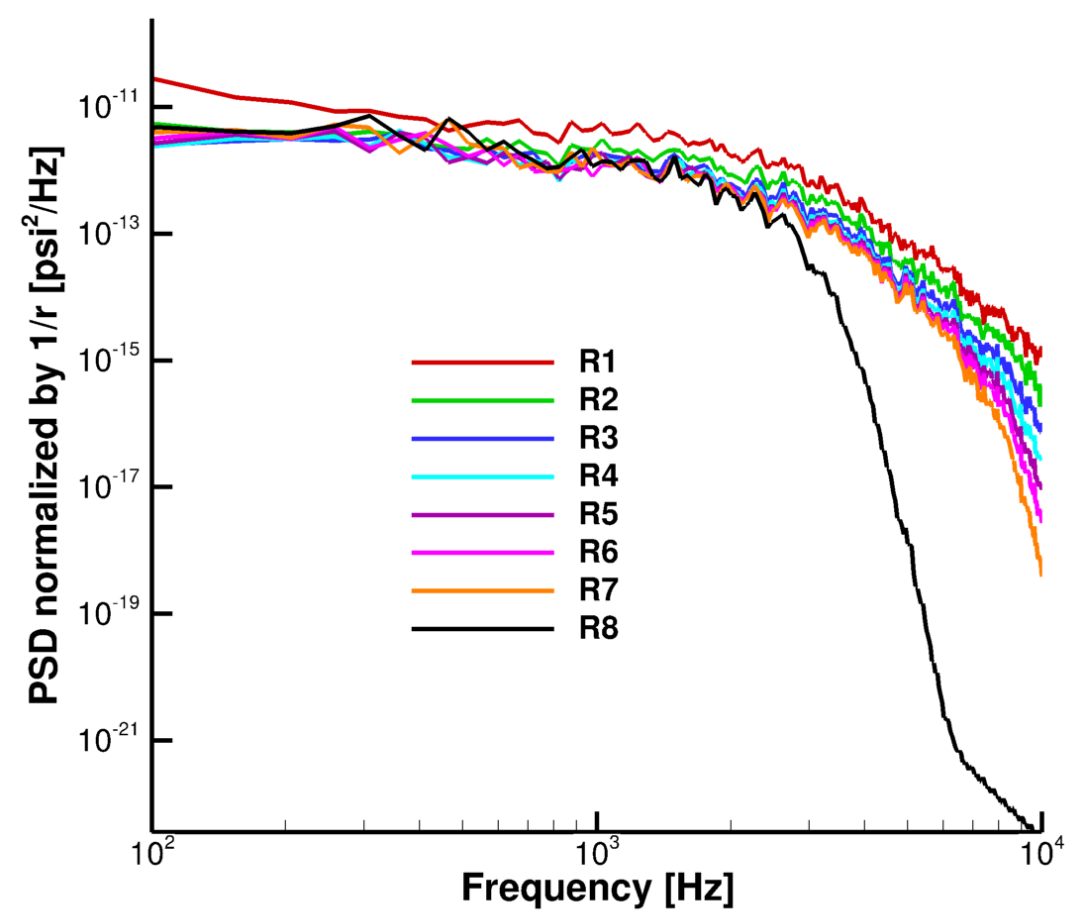

Figure 12. PSD normalized by radial distance for points in $\mathbf{y}=\mathbf{0}$ plane.

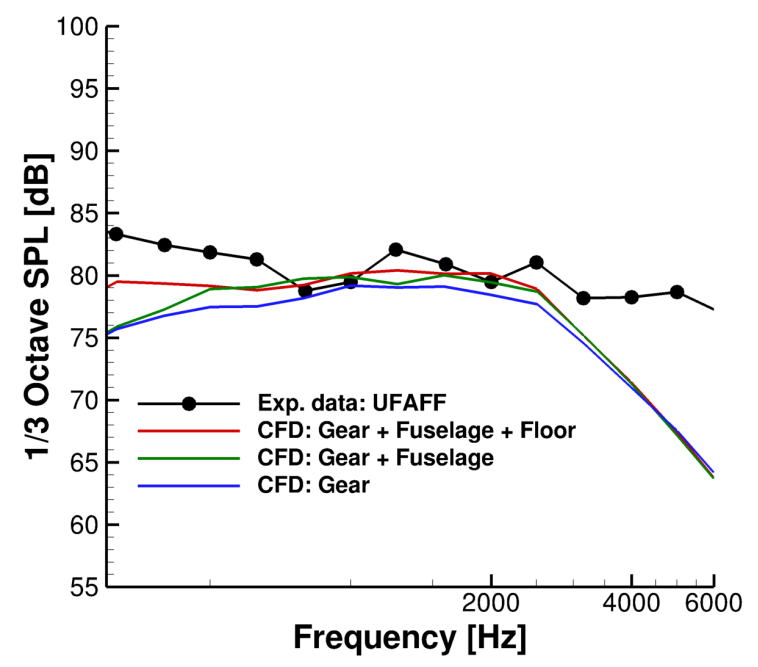

(a) UFAFF simulations

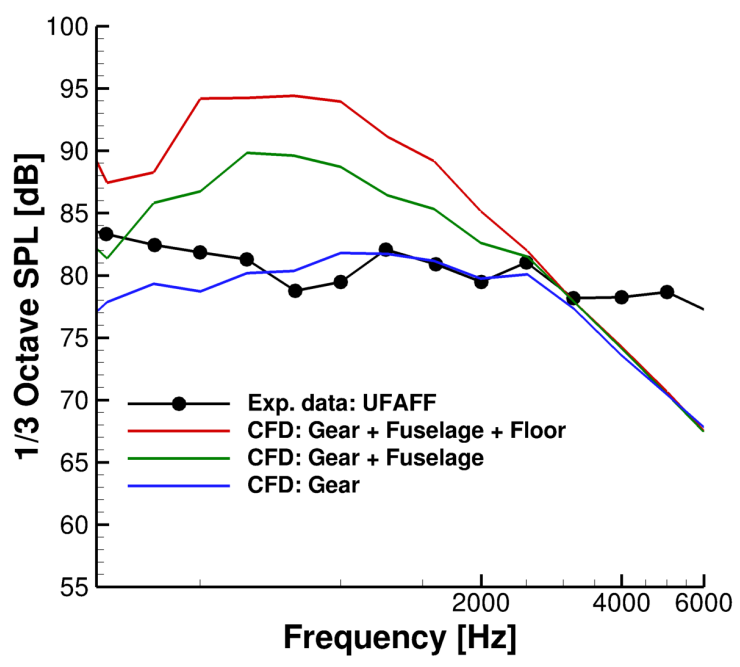

(b) BART simulations

Figure 13. Sound pressure level comparisons at flyover mic 4 using solid surface perturbations. 


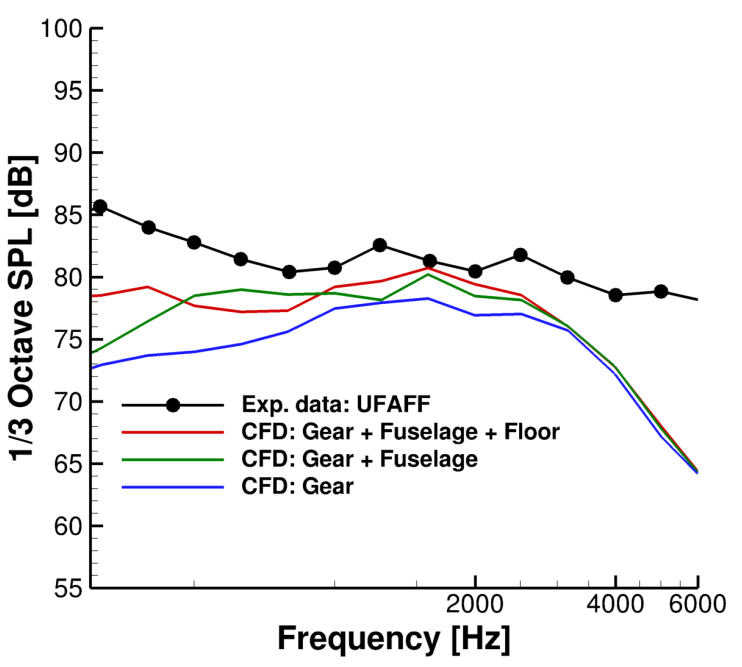

(a) UFAFF simulations

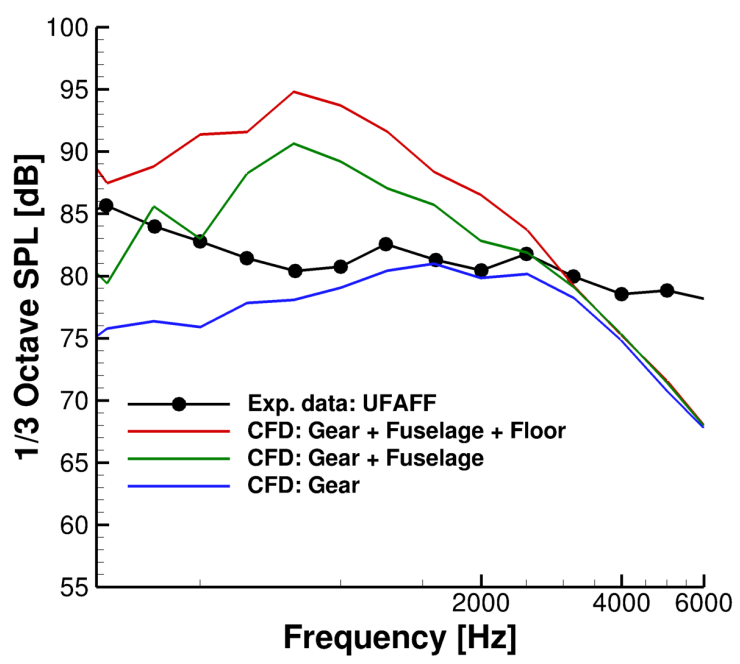

(b) BART simulations

Figure 14. Sound pressure level comparisons at flyover mic 7 using solid surface perturbations.

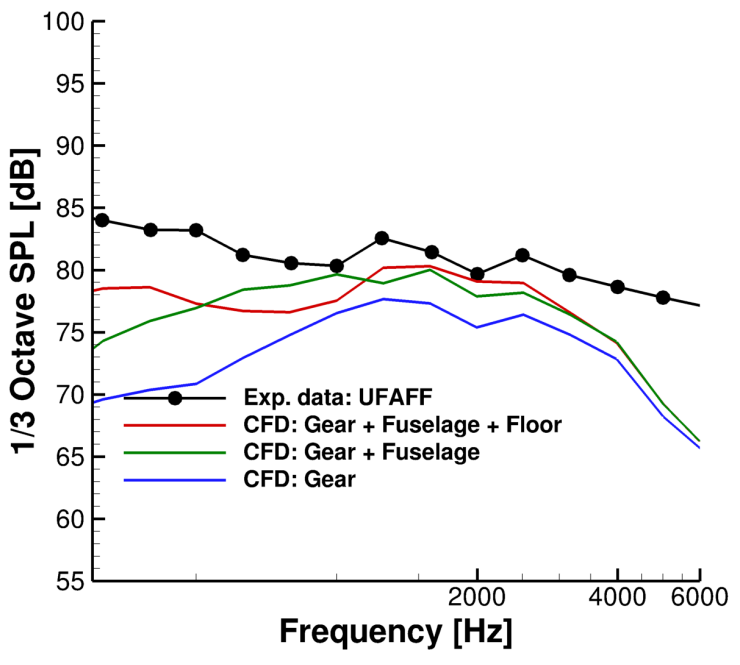

(a) UFAFF simulations

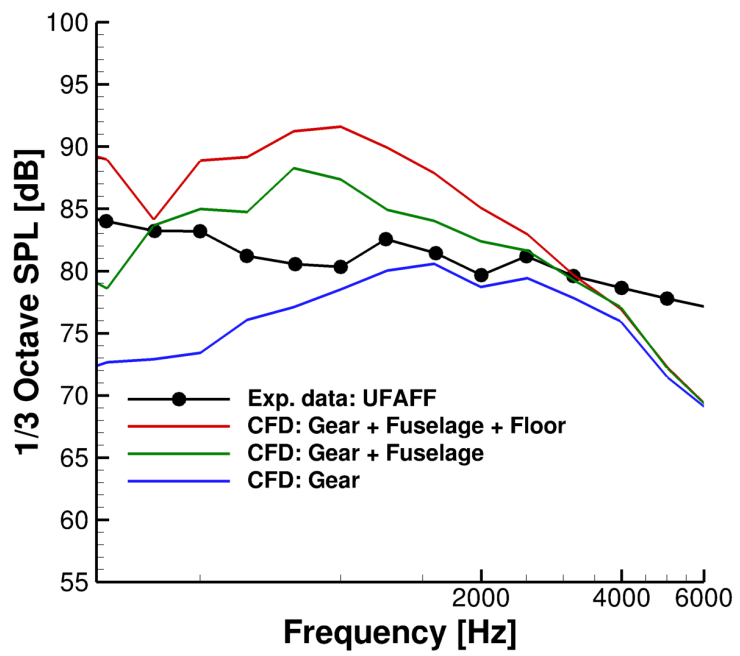

(b) BART simulations

Figure 15. Sound pressure level comparisons at flyover mic 9 using solid surface perturbations.
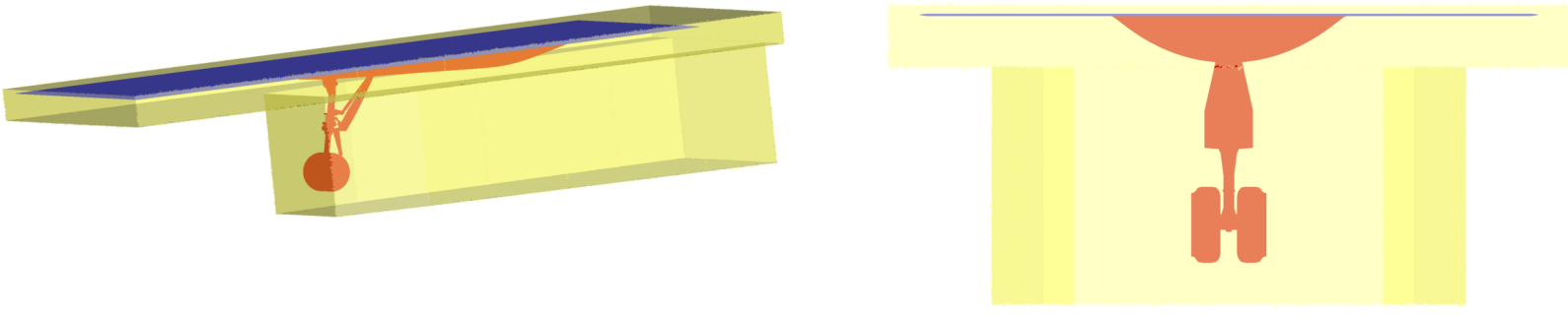

(a) Perspective view

(b) Front view

Figure 16. Schematic views of permeable surface used for noise prediction. 


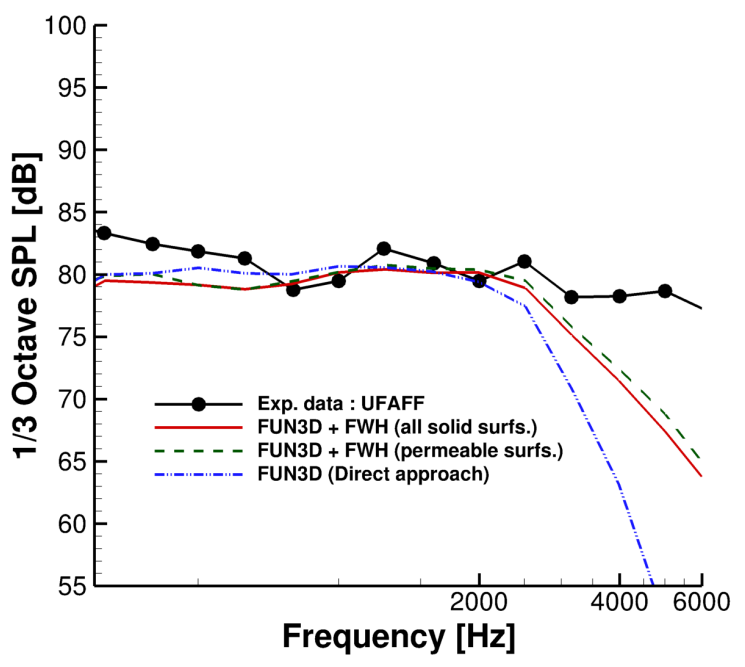

(a) Flyover mic 4

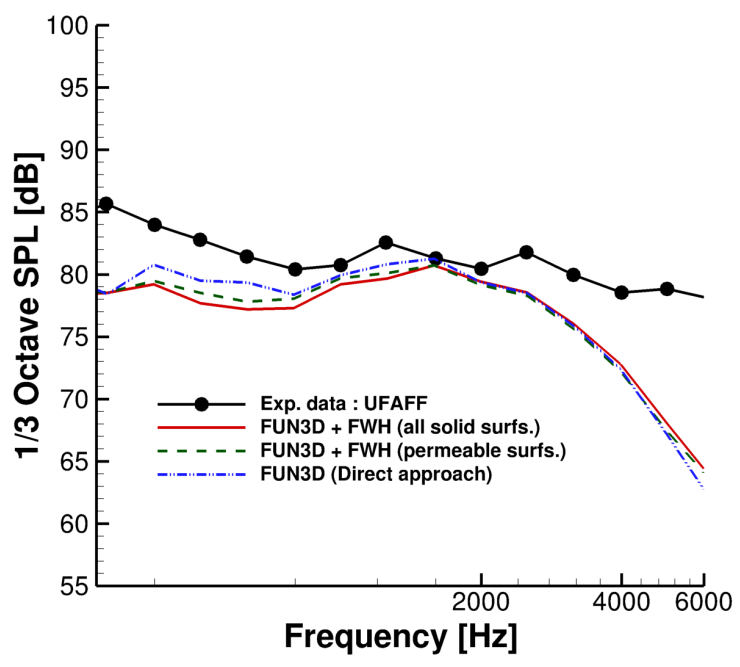

(b) Flyover mic 7

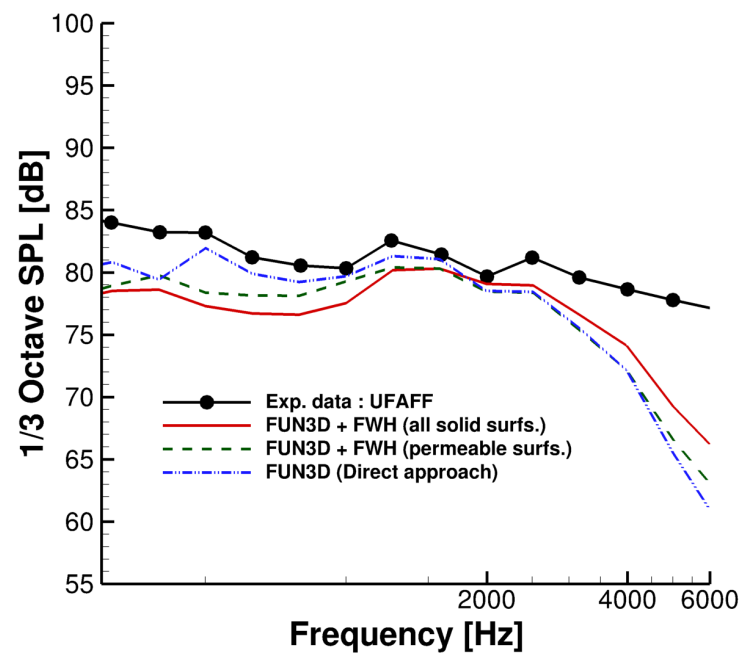

(c) Flyover mic 9

Figure 17. Sound pressure level comparisons for UFAFF configuration. 